\title{
Analysis of isoplanatic high resolution stellar fields by the StarFinder code
}

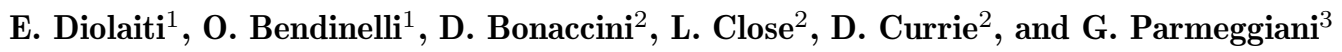 \\ 1 Universitá di Bologna, Dipartimento di Astronomia, via Ranzani 1, I-40127 Bologna, Italy \\ 2 European Southern Observatory, Karl-Schwarzschild Str. 2, D-85748 Garching, Germany \\ 3 Osservatorio Astronomico di Bologna, via Ranzani 1, I-40127 Bologna, Italy
}

Received June 20; accepted September 8, 2000

\begin{abstract}
We describe a new code for the deep analysis of stellar fields, designed for Adaptive Optics (AO) Nyquistsampled images with high and low Strehl ratio. The Point Spread Function (PSF) is extracted directly from the image frame, to take into account the actual structure of the instrumental response and the atmospheric effects. The code is written in IDL language and organized in the form of a self-contained widget-based application, provided with a series of tools for data visualization and analysis. A description of the method and some applications to AO data are presented.
\end{abstract}

Key words: instrumentation: adaptive optics - method: data analysis - techniques: image processing techniques: photometric — astrometry — stars: imaging

\section{Introduction}

StarFinder is a code developed within the frame of the ESO PAPAO (Currie et al. 2000a, 2000b) program for the reduction of Adaptive Optics data. It was originally designed (Diolaiti et al. 1999) to analyze AO images of very crowded fields, like the PUEO frame of the Galactic Center shown in this paper, which contains about 1000 detectable stars in a field of view of $13 \times 13 \operatorname{arcsec}^{2}$. This image is an example of an AO observation: the PSF is Nyquist-sampled and characterized by a complex shape, showing a sharp peak, one or more fragmented diffraction rings and an extended irregular halo. Moreover, due to the small field of view, the imaged region is approximately isoplanatic and the PSF may be considered space invariant. Under the assumptions of isoplanatism and Nyquistsampling, StarFinder models the observed stellar field as

Send offprint requests to: E. Diolaiti,

e-mail: diolaiti@bo.astro.it a superposition of shifted scaled replicas of the PSF lying on a smooth background due to faint undetected stars, possible faint diffuse objects and noise.

The procedure derives first a PSF digital template from the brightest isolated field stars; then a catalogue of presumed objects is formed, searching for the relative intensity maxima in the image frame. In the following step the images of the suspected stars are analyzed in order of decreasing luminosity. In this phase a catalogue including the accepted objects is formed and a synthetic image of the observed field is constructed, placing an intensityscaled PSF template in the position of each identified star. Each suspected object in the original list is accepted on the basis of its correlation coefficient with the PSF template; the relative astrometry and photometry of the source are determined by means of a fit, taking into account the contribution of the local non-uniform background and of the already detected stars, known from the synthetic field. As the analysis proceeds, fainter and fainter sources can be successfully analyzed, discriminating their central peaks from the secondary bumps of light in the distorted diffraction rings of the neighboring more luminous already identified stars: in this way the synthetic field becomes more and more similar to the observed image. Residual unexplained features of the image may be further analyzed checking for indications of blended groups, at separations smaller than the PSF FWHM.

StarFinder should be regarded as a tool for high precision astrometry and photometry of crowded fields acquired under the above assumptions of accurate PSF knowledge, isoplanatism and correct sampling. An application of StarFinder to HST undersampled data handled by dithering techniques (Fruchter et al. 1997) has been shown in Aloisi et al. (2000). In this paper it can be seen that the results obtained by our method are comparable to those obtained by DAOPHOT (Stetson 1987).

As far as the PSF is concerned, the accurate knowledge of its features outside the central peak is fundamental 
to perform a deep study of a stellar field, achieving accurate photometry of faint stars and avoiding either false detections or star loss (Esslinger \& Edmunds 1998). If the PSF template cannot be extracted directly from the field, due to extreme crowding or lack of bright isolated stars, StarFinder can still be applied using a PSF estimated by means of other methods, as the reconstruction technique proposed by Véran et al. (1997) for AO observations or the TINY TIM simulation software for HST (Krist \& Hook 1999).

Much more intriguing and difficult to solve is the case of a field with space variant PSF, either due to anisoplanatic effects as in AO observations or to design and control in HST images. In general the analysis of an anisoplanatic field requires the knowledge of the local PSF. A method to reconstruct the off-axis PSF in AO imaging has been proposed by Fusco et al. (2000). StarFinder, in its present version, can analyze frames with space invariant PSF or sub-frames in which the isoplanicity condition is nearly satisfied, as will be shown in Sect. 4; the extension to the space variant case is in progress, with preliminary results presented by Diolaiti et al. (2000a, 2000b).

The paper is organized as follows: the general features of the algorithm are described in Sect. 2; Sect. 3 deals with more technical aspects and might be skipped on a first reading; the method is validated on simulated and experimental data in Sect. 4; details on the IDL code are presented in Sect. 5; Sect. 6 includes our conclusions and future plans.

\section{Code description}

\subsection{PSF estimation}

The accuracy of the PSF estimate is primary in StarFinder, since the PSF array is used as a template for all the stars in the image to be analyzed. The user selects the most suitable stars, which are background subtracted, cleaned from the most contaminating sources around, centered with sub-pixel accuracy, normalized and superposed with a median operation. The centering is performed by an iterative shift of the stellar image in order to cancel the sub-pixel offset of its centroid (see Christou \& Bonaccini 1996). The median operation, which is performed pixel-by-pixel, is preferred to the mean because it is less sensitive to anomalous pixels (outliers) which might appear in one or more stellar images among the selected ones. The retrieved PSF is post-processed in order to reject any residual spurious feature and to smooth the noise in the extended halo.

The PSF extraction procedure also reconstructs the core of saturated stars by replacing the corrupted pixels with the central part of the PSF estimate. Accurate positioning is achieved by means of a cross-correlation technique, while the scaling factor is determined with a least squares fit to the wings of the star to repair. For a detailed description of the procedure, see Sect. 3.5.

\subsection{Standard analysis of a stellar field}

At first we build a list of objects, the presumed stars, which satisfy the condition

$i(x, y)>b(x, y)+t$

where $i(x, y)$ is the observed intensity, $b(x, y)$ the background emission and $t$ a fair detection threshold. The presumed stars are analyzed one by one by decreasing intensity. To illustrate a generic step of the analysis, we consider the $(n+1)$-th object in the list, after the examination of the first $n$. A small sub-image of fixed size is extracted around the object (Fig. 1, left). This sub-image may contain brighter stars formerly analyzed, fainter objects neglected in the current step and features of other stars lying outside the sub-image. The information on the brighter sources is recorded in a synthetic stellar field (Fig. 1, right), defined as the sum of two terms: a superposition of PSF replicas, one for each star detected up to this point, and an estimate of the background, assumed to be non uniform in general. The local contribution due to the brighter stars and the background, derived from the synthetic field, is subtracted from the sub-image. If a statistically significant residual remains, it is compared to the central core of the PSF by means of a correlation check. If the correlation coefficient is higher than a prefixed threshold then the object of interest is rated similar to the PSF and accepted. The accurate determination of its position and relative flux is attained by means of a local fit, in which the observed sub-image is approximated with the multi-component model described in Sect. 3.6. The actual size of the fitting region is comparable to the diameter of the first diffraction ring of the PSF. This choice ensures that the information represented by the shape of a highStrehl PSF is included in the fitting process to achieve better accuracy and prevents the growth of the number of sources to be fitted together. For the central object of our example a single component fit is performed and the contribution due to the brighter stars is considered as a fixed additive term. A multi-component fit is performed when the star is in a very compact group, at separations comparable to the PSF FWHM. If the fit is acceptable, the parameters of the new detected star are saved and those of the already known sources, which have been possibly re-fitted, are upgraded. The new star and an upgrade of the re-fitted sources are added to the synthetic field.

This analysis is performed for each object in the initial list (a flow-chart illustrating the operations of StarFinder is in Fig. 2). To achieve a better astrometric and photometric accuracy, at the end of the examination of all the objects, the detected stars are fitted again, this time considering all the known sources. This step may be iterated 


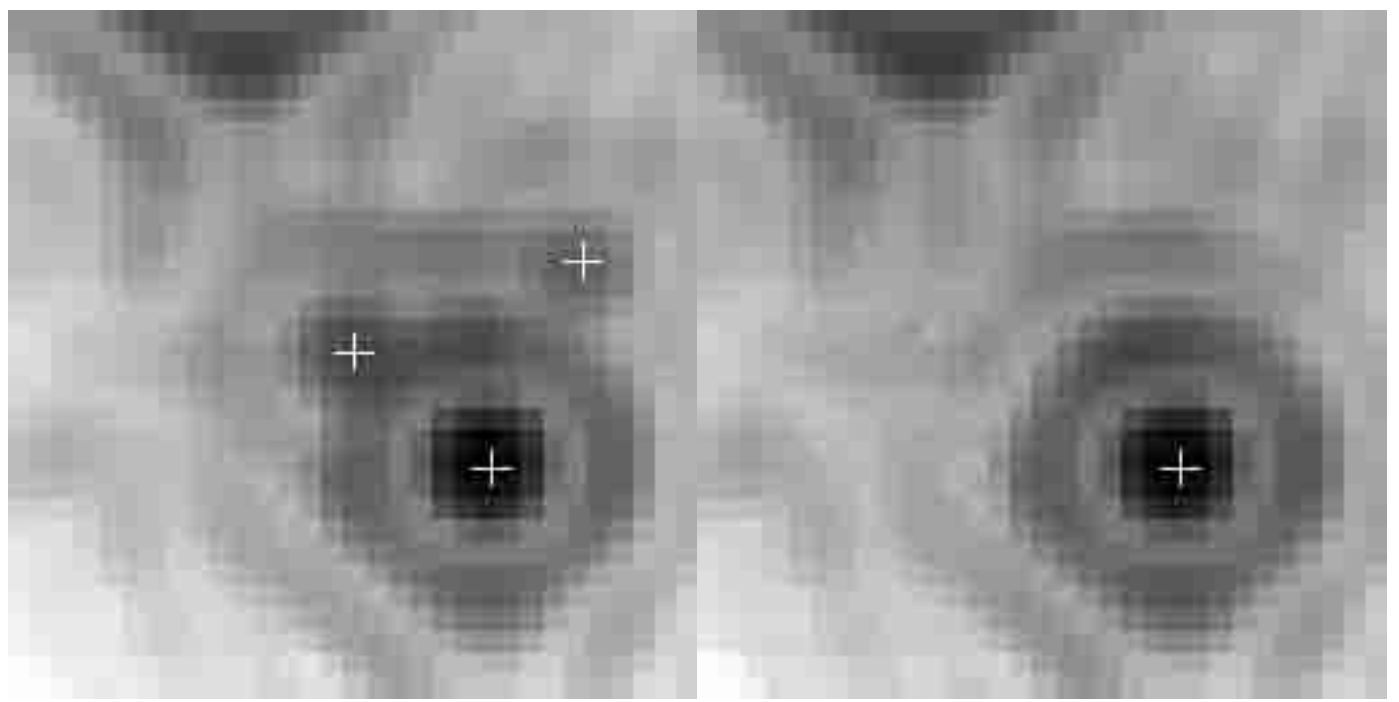

Fig. 1. Left: sub-image extracted from a simulated stellar field. The crosses indicate the objects within the region of interest: a central star (to be analyzed in the current step), a brighter source (already known), a fainter one (to be examined later) and the PSF feature of a much brighter star, represented by the structure in the upper-left part of the sub-image. Right: corresponding sub-region extracted from the stellar field model, containing one replica of the PSF for each star detected so far

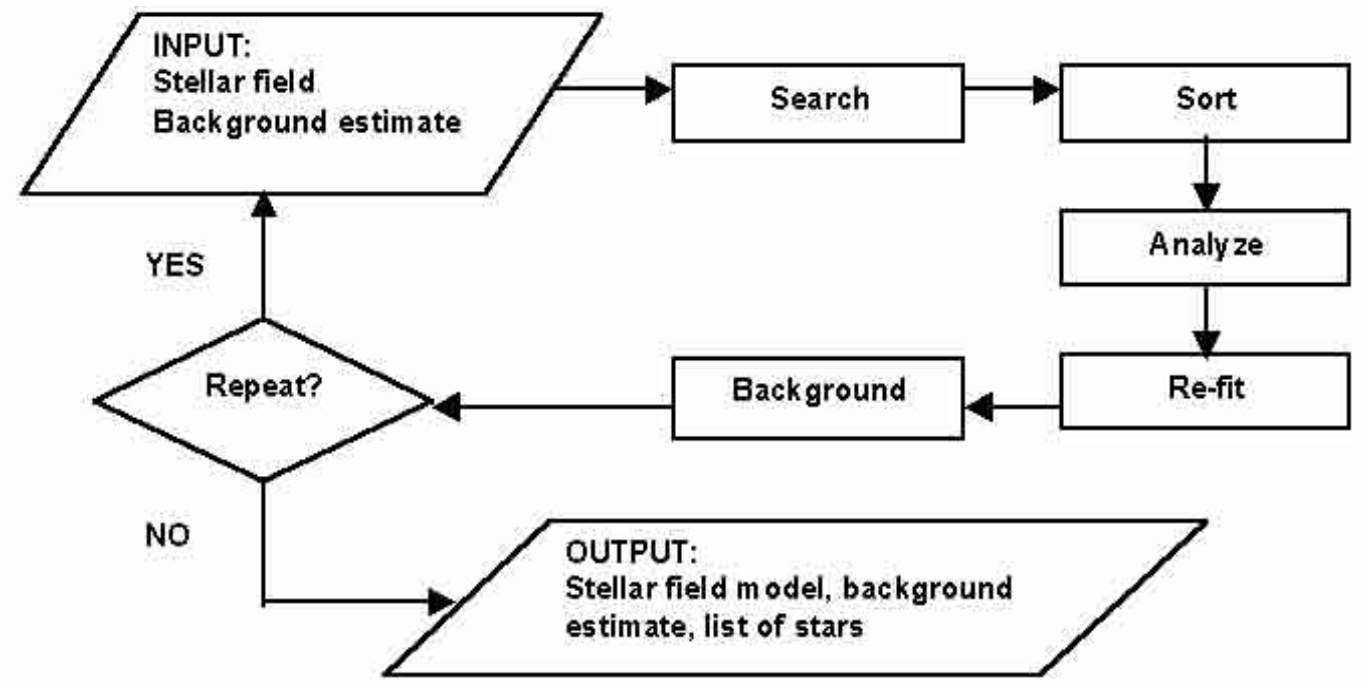

Fig. 2. Flow-chart of the algorithm for stars detection and analysis

a pre-fixed number of times or until a convergence condition is met.

At the end of the analysis, it is possible to stop the algorithm or instead perform a new search for lost objects removing the detected stars and using an upgraded background estimate. It should be stressed that this image subtraction is just a tool to highlight significant residuals. Any further analysis is performed on the original frame, in order to take into account the effects arising from the superposition of the PSFs of neighboring sources. Generally, after 2-3 iterations of the main loop, the number of detected stars approaches a stable value.

\subsection{Crowding and blending effects}

A binary star with different separation values (Fig. 3) has been simulated to show how the code works with crowded sources. With a separation of 2 PSF FWHM the two components are well separated and the code analyzes them with the standard procedure described in the previous sub-section. In the other cases (separation from 1 to 0.5 PSF FWHM) the secondary component is not detected as a separate relative maximum and it is lost. However, if the separation is not as small as 0.5 FWHM, a further iteration of the main loop enables the algorithm to detect the fainter component by subtracting the brighter one. 


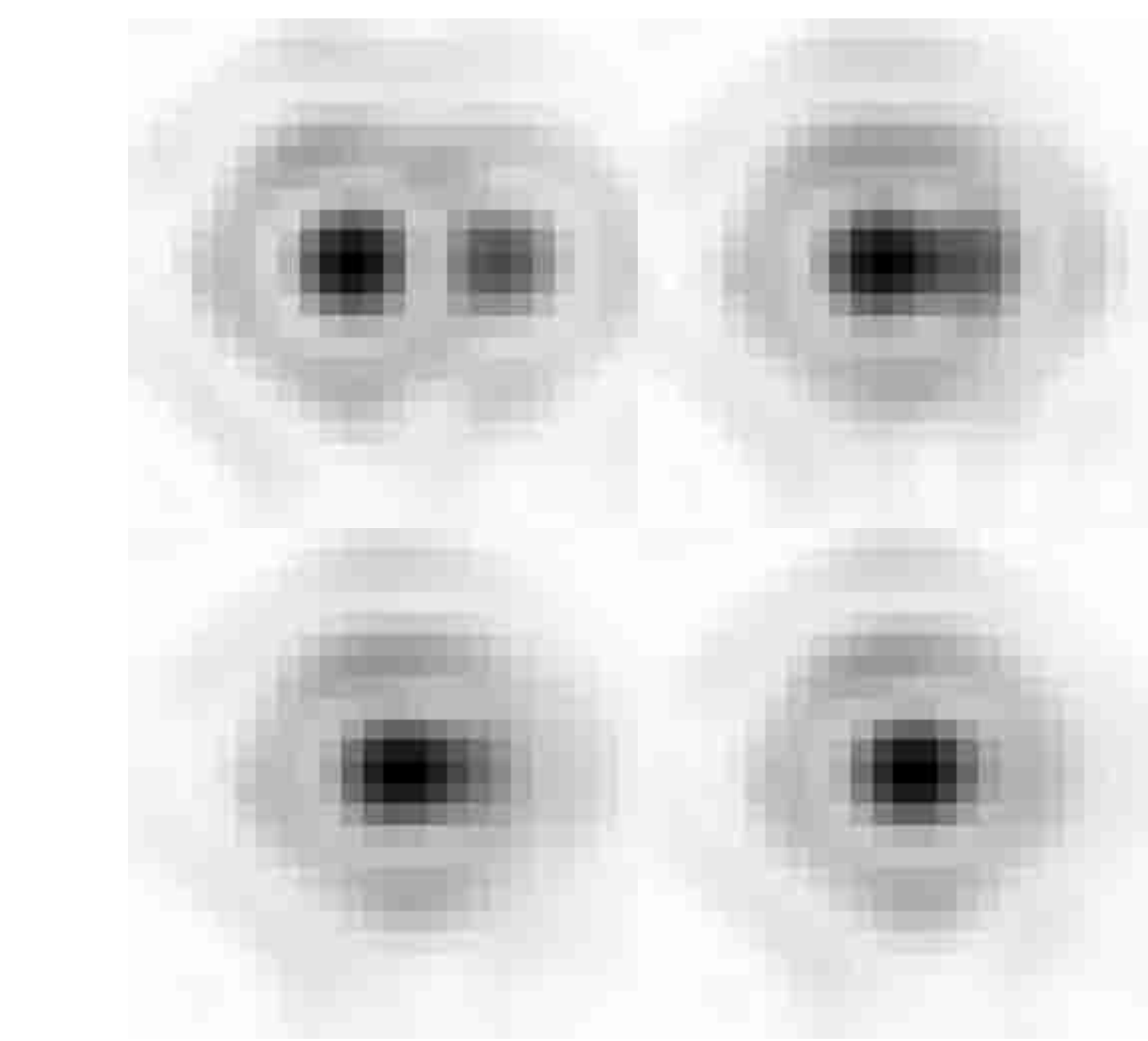

Fig. 3. Simulated binary stars at various separations. From left to right, top to bottom the separation is $2,1,0.75,0.5$ times the PSF FWHM. For all the images the flux ratio is $2: 1$

This strategy forces the two stars to pass the correlation test, the principal component as a presumed single object and the secondary in a subsequent iteration of the loop. In a way the iteration of the main loop is a de-blending strategy, because it enables the algorithm to detect stars whose intensity peak is not directly visible in the observed data. This strategy fails when:

- the binary is very noisy and the two components have similar flux. In a similar situation the principal component may not pass the correlation test, preventing also the detection of the secondary in a further iteration. These noisy blends may be recovered at the end of the overall analysis;

- The two components are almost equally bright and have a separation close to the lower limit $(1 / 2 \mathrm{PSF}$ FWHM). The residual corresponding to the secondary after subtracting the principal component may have a distorted shape and might not pass the correlation check.
The latter case may be handled by a method based on a thresholding technique. The object is cut at a prefixed level, about $20 \%$ below the central peak, and transformed to a binary array, setting to 1 all the pixel above the threshold and to 0 the pixels below. If the area of the pixels with value 1 is more extended than the PSF, the object is considered a blend and the secondary star may be detected by subtracting the brighter one; then a twocomponent fit allows accurate astrometry and photometry of the two sources. This strategy can be iteratively applied to multiple blends. It should be stressed that the area measurement is not reliable when the value of the cutting threshold is comparable to the noise level: for this reason the de-blending procedure is applied only to objects with a suitable signal-to-noise ratio. Moreover the area measurement is reliable when the data are adequately sampled. This de-blending procedure is applied at the end of the last iteration of the main loop, when all the resolved sources have been detected: in this way the probability that a single object may appear artificially blurred because of 
the contamination of still unknown sources is largely reduced.

In a normal case, like the simulated field of Sect. 4.1, two or three iterations of the main loop find almost all $(\sim 99 \%)$ the stars that StarFinder may detect. The deblending procedure described above adds $\sim 1 \%$ more stars, without additional false detection. The number of lost objects belonging to the first category described above is negligible $(\ll 1 \%)$.

Normally we perform two or three iterations of the main loop and apply the de-blending strategy only in very crowded fields.

\section{Technical aspects}

\subsection{Bad pixels}

StarFinder includes an automatic procedure to repair known bad pixels, which are replaced with the median of the good data points in a suitable neighborhood. The use of this procedure is particularly recommended in the extraction of the PSF. Indeed the stellar images, which will be combined to form the PSF, are centered with sub-pixel accuracy by means of an interpolation technique and the presence of bad pixels may produce unpredictable interpolation errors. Even if replaced, the bad pixels are excluded from any further computation concerning the correlation coefficient and the fitting process.

\subsection{Background estimation}

A reliable estimate of the background is necessary to define the detection condition and to compute the correlation coefficient of the presumed stars with the PSF.

A straightforward technique to estimate the background is to smooth the image by median filtering, replacing each pixel with the median computed over a suitable neighborhood, of size comparable to the characteristic width of a stellar image. This method tends to over-estimate the background underneath strong peaks. A more accurate approximation, even in crowded fields, is described in Bertin \& Arnouts (1996). The image is partitioned in sub-regions arranged in a regular grid and a local estimate is calculated for each sub-patch by means of an IDL implementation of the DAOPHOT SKY routine (Stetson 1987), due to Landsman (1995). This array of sky measurements is smoothed by median filtering and interpolated onto the same grid of the input image.

It should be stressed that the background computation is unavoidably affected by the presence of bright sources. In general a more accurate estimate can be obtained after the analysis of the stellar field, when the most contaminating sources are known and can be subtracted.

\subsection{Noise estimation}

The estimate of the noise is useful to define the detection threshold and to compute the formal errors on the retrieved astrometry and photometry.

The overall effect of photon and instrumental noise can be computed if the required parameters (detector gain, read-out-noise, dark current, etc.) are known. Otherwise an estimate of the mean background noise can still be obtained by means of histogram fitting techniques (Almoznino et al. 1993; Bijaoui 1980). Assuming that the intensity of the sky radiation is distributed normally around a typical value, the histogram of the observed intensity levels should be quite similar to a Gaussian distribution, whose mode and standard deviation represent respectively the sky value and the associated noise. Actually the contamination due to the stellar sources produces a high-intensity tail and an artificial broadening of the histogram, which prevents an accurate estimate of the background noise. This problem can be partially overcome by removing the signal from the image, leaving only the pixel-to-pixel variations associated to pure noise: a reasonable estimate of the signal to subtract for this purpose can be obtained by smoothing the data with a median filtering technique. After this operation the histogram is symmetric around its mode and the background noise standard deviation can be estimated by means of a Gaussian fit to the histogram itself.

\subsection{Correlation coefficient}

False detections, associated to noise spikes or residual PSF features of bright stars, are recognized on the basis of their low correlation coefficient with the PSF, which represents a template for each true star in the image. The correlation coefficient (Gonzalez \& Woods 1992) is defined as

$c(a, b)=$

$$
\frac{\sum_{x, y}[i(x, y)-\bar{\imath}][p(x-a, y-b)-\bar{p}]}{\sqrt{\sum_{x, y}[i(x, y)-\bar{\imath}]^{2}} \sqrt{\sum_{x, y}[p(x-a, y-b)-\bar{p}]^{2}}}
$$

where $i(x, y)$ and $p(x, y)$ are the object and the PSF respectively, $\bar{\imath}$ and $\bar{p}$ are the corresponding mean values. Maximizing the correlation coefficient as a function of the offset $(a, b)$ yields an objective measure of similarity. After maximizing $c(a, b)$ for integral offsets, it is possible to repeat the procedure for sub-pixel shifts, improving the positioning accuracy.

The correlation coefficient is computed on the core of the PSF: typically the central spike of the diffraction pattern is considered, out to the first dark ring. A fair correlation threshold must be fixed in order to discriminate and reject unlikely detections, without losing faint stars contaminated by the background noise; a value of 0.7 or 0.8 is acceptable in most cases. 
The correlation coefficient represents also an effective tool to select the stars with the highest photometric reliability, since generally a very high correlation value is associated to resolved single sources.

\subsection{Saturated stars}

Saturated stars provide precious information on the PSF halo, so it may be useful to include them, appropriately reconstructed, in the PSF extraction process. In addition, the repaired saturated stars can be recognized by the star detection algorithm and their contribution be taken into account during the analysis of near and fainter sources.

The core of a saturated star is replaced with a shifted scaled replica of a preliminary estimate of the PSF. The repaired star is defined as

$i_{\text {rep. }}(x, y)= \begin{cases}i(x, y) & \text { if } i(x, y)<T \\ f p\left(x-x_{0}, y-y_{0}\right) & \text { otherwise }\end{cases}$

where $T$ is the upper linearity threshold of the detector. The position of the center $\left(x_{0}, y_{0}\right)$ is estimated by means of the maximization of the correlation coefficient, which is not sensitive to the intensity levels. The scaling factor $f$ is calculated with a least squares fit to the wings of the saturated star. Of course the saturated pixels are excluded from all the computations. The background is temporarily subtracted in order to prevent affecting the repair process.

This procedure has been applied to the brightest star (IRS 7) of the Galactic Center image shown in Sect. 4.2. This source is not saturated in the original data, but it has been artificially corrupted by an upper cut at half maximum. The repair procedure is able to reconstruct the original peak with an error of $\sim 5 \%$, imposing a positioning accuracy of $1 / 2$ pixel.

\subsection{Fitting procedure}

A sub-image centered on the star of interest is extracted and approximated with the model

$$
\begin{aligned}
h(x, y)= & s_{0}(x, y)+\sum_{n=1}^{N_{\mathrm{s}}} f_{n} p\left(x-x_{n}, y-y_{n}\right) \\
& +b_{0}+b_{1} x+b_{2} y
\end{aligned}
$$

where $s_{0}(x, y)$ is the fixed contribution of known stars outside the sub-image support, $N_{\mathrm{s}}$ is the number of point sources within the sub-image, $x_{n}, y_{n}, f_{n}$ are the position and flux of the $n$-th source, $p(x, y)$ is the PSF and $b_{0}, b_{1}$, $b_{2}$ are the coefficients of a slanting plane representing the local background. It should be stressed that the retrieved astrometry and photometry are referred to the absolute centering and normalization of the PSF array. The optimization of the parameters is performed by minimizing the least squares error between the data and the model. If the noise is known, it is possible to weigh the data by their inverse standard deviation and obtain a statistically optimal fit (Beck \& Arnold 1977). In this case, formal error estimates on the parameters can be obtained (Bevington \& Robinson 1994). The optimization is performed by means of an iterative Newton-Gauss technique with linearized Hessian (Beck \& Arnold 1977; Luenberger 1984). The Moore-Penrose generalized inverse concept (Rust \& Burrus 1972; Bendinelli et al. 1987; Lorenzutta 1967) is applied to invert the Hessian matrix at every step. The iterations are stopped when the parameters approach a stable value. The major difficulty of a Newton-like method is represented by the computation of the model derivatives with respect to the parameters, some of which (stellar positions) yield non-linear dependence. For this purpose the Fourier shift theorem is applied:

$$
\begin{aligned}
& p\left(x-x_{n}, y-y_{n}\right)= \\
& \mathrm{FT}^{-1}\left[\mathrm{FT}[p(x, y)] \mathrm{e}^{-i 2 \pi\left(u x_{n}+v y_{n}\right) / N}\right]
\end{aligned}
$$

where FT represents the discrete Fourier transform operation, $N$ is the sub-image size and $u, v$ are spatial frequencies. The derivative with respect to $x_{n}$ is then

$$
\begin{aligned}
& \frac{\partial p\left(x-x_{n}, y-y_{n}\right)}{\partial x_{n}}= \\
& \mathrm{FT}^{-1}\left[\mathrm{FT}[p(x, y)] \mathrm{e}^{-i 2 \pi\left(u x_{n}+v y_{n}\right) / N}\left(-i \frac{2 \pi u}{N}\right)\right]= \\
& \mathrm{FT}^{-1}\left[\left(-i \frac{2 \pi u}{N}\right) \operatorname{FT}\left[p\left(x-x_{n}, y-y_{n}\right)\right]\right]
\end{aligned}
$$

and requires in practice an interpolation of the PSF to compute $p\left(x-x_{n}, y-y_{n}\right)$. In principle this interpolationbased method can only be applied to Nyquist-sampled images and this is currently the main limit of the algorithm. A similar technique has been described by Véran \& Rigaut (1998).

\subsection{Sampling and interpolation}

A band-limited function in one dimension is Nyquistsampled if the step size fulfills the condition

$\Delta x \leq \frac{1}{2} f_{\mathrm{c}}$

where $f_{\mathrm{c}}$ is the so-called cut-off frequency of the spectrum. In this case it is possible to reconstruct the original continuous function from a set of equally-spaced samples by means of the so-called sinc interpolation (Mariotti 1988). For a nearly diffraction-limited PSF, the critical sampling condition is generally stated by saying that its FWHM must contain at least two pixels. Many accurate and efficient interpolation schemes exist to perform the PSF shift required by the fitting procedure. Probably the most simple is a straightforward application of the Fourier shift theorem, but one may also use bicubic splines or the fast sinc interpolation algorithm described in Yaroslavksy (1997). In StarFinder we have applied the cubic convolution interpolation method (Park \& Schowengerdt 1983), which 


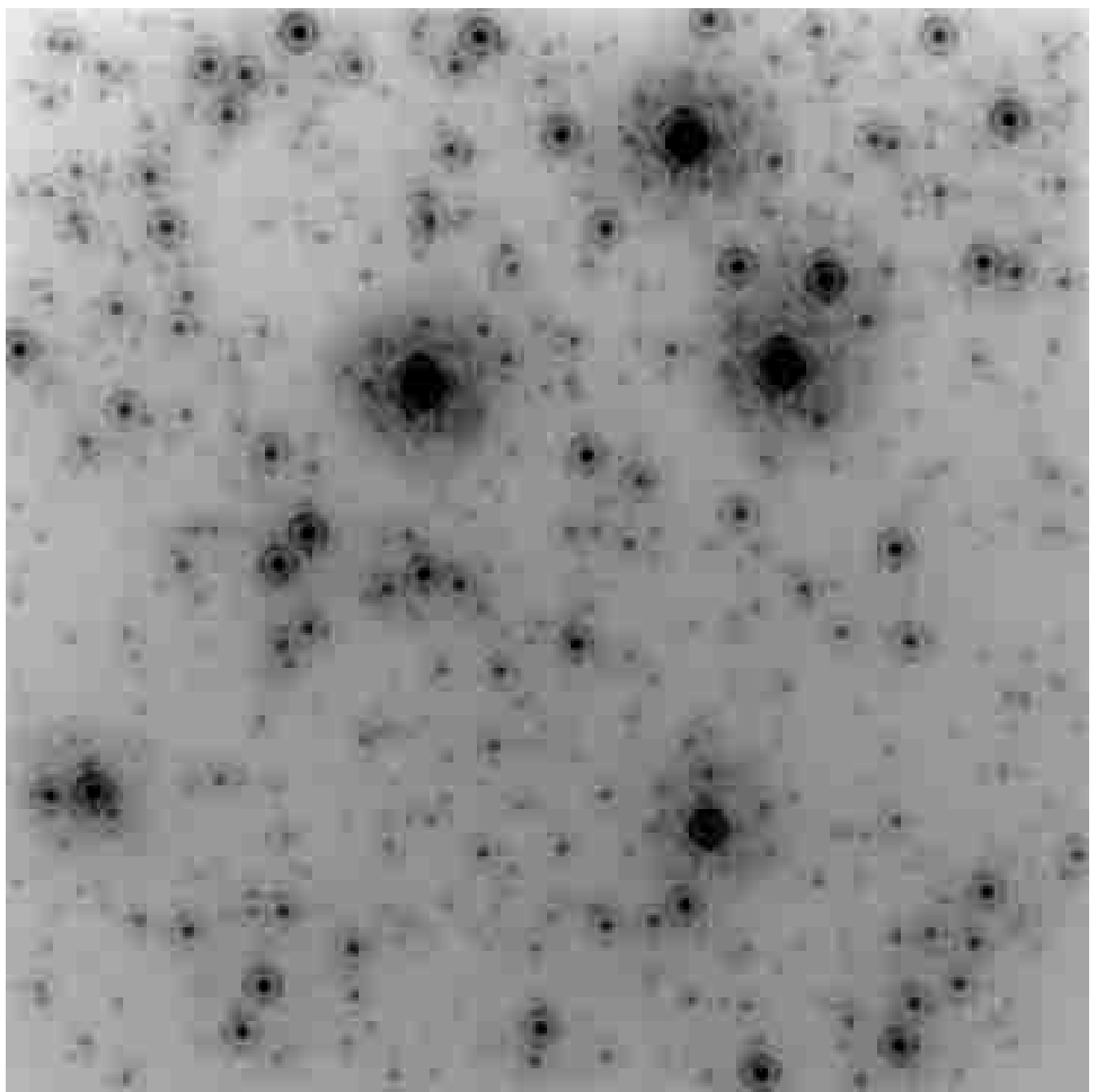

Fig. 4. Synthetic field image with 1000 sources. The PSF Strehl ratio is $\sim 40 \%$. The display stretch is logarithmic

approaches very closely the optimal sinc interpolation for Nyquist-sampled data; this algorithm is implemented in the IDL function INTERPOLATE.

Even though the interpolation of the PSF array is allowed only on Nyquist-sampled data, the cubic convolution technique seems to produce acceptable results even with marginally under-sampled images. Tests performed on Airy diffraction patterns, with a sampling step twice as large as the critical sampling step size, indicate that the interpolation-induced oscillations amount to a few percent of the image peak, as opposed to $10-20 \%$ of other interpolation techniques like Fourier shift or bicubic splines.

\section{Applications}

\subsection{Simulated field}

The code was first applied to a simulated image (Fig. 4) including 1000 stars, placed randomly in the frame and with a given magnitude distribution (Fig. 6). Each star is a scaled copy of a long exposure high-Strehl PSF, obtained with the ADONIS AO system at the ESO $3.6 \mathrm{~m}$ telescope. The image is $368 \times 368$ pixels large $\left(13^{\prime \prime} \times 13^{\prime \prime}\right)$ and has a stellar density of 6 stars $\operatorname{arcsec}^{-2}$. Photon, readout noise and a background nebulosity, normalized to the same flux of the stellar sources, were added to the image. The faintest stars have a peak signal-to-noise ratio of 5 .

We performed a standard reduction of the artificial image using the "default" parameters of the method.

The PSF was estimated by superposing the images of the four brightest stars in the field. The retrieved PSF is very similar to the true one (Fig. 5).

Figure 6 shows the good agreement between the true and the observed luminosity function. The sole statistically significant discrepancy is in the bin from magnitude 7 to 8 , where $\sim 25 \%$ of the stars were lost; the other small differences are due to photometric errors which shift some objects to a neighboring magnitude interval. The lost stars are $\sim 10 \%$ of the total number of sources and are generally faint: about $90 \%$ have magnitude between 7 and 8 , the rest is in the bin between magnitude 6 and 7 . The only 


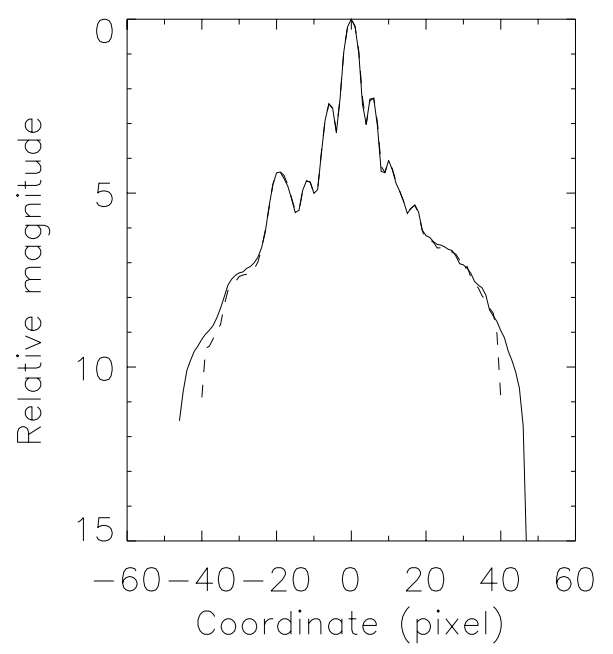

Fig. 5. Axial plot of the true PSF (continuous line) and of the retrieved PSF (dashed line)

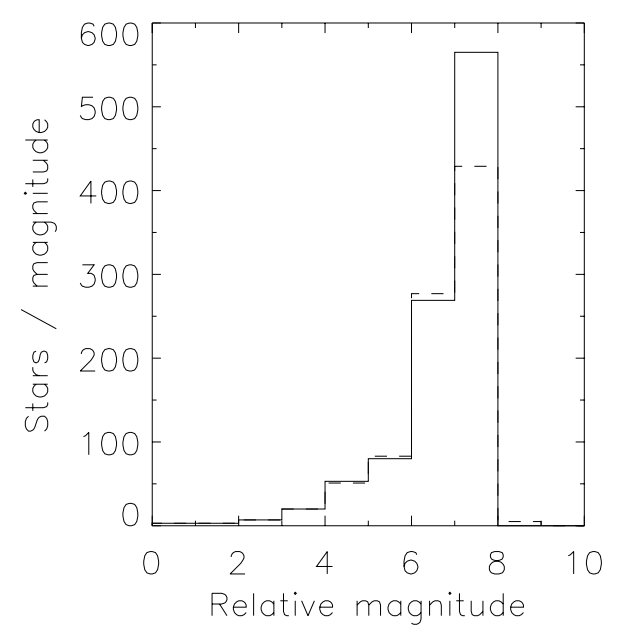

Fig. 6. Comparison between the true (continuous line) and the estimated luminosity function (dashed line)

bright lost star has magnitude $\sim 4.5$ and is the secondary component of a very close binary, with a separation of just $1 / 2$ pixel. Roughly $70 \%$ of the lost stars are located at a distance $\leq 1$ PSF FWHM from the nearest object, $\sim 20 \%$ are on the first diffraction ring of a brighter source and only $\sim 10 \%$ are isolated. It should be stressed however that $\sim 15 \%$ of the lost stars can be recognized by visual inspection as faint objects in the halo of the four brightest stars in the field, independently of their separation from the nearest source. It is apparent that the blending effect and the contamination by the halo of very bright stars do account for the lost stars. Note that the number of false detections in this simulated field is negligible (1 case out of 1000).

The plots in Figs. 7 and 8 show the astrometric and photometric accuracy of StarFinder. About $80 \%$ of the detected stars have both astrometric error smaller than 0.1 PSF FWHM and photometric error smaller than

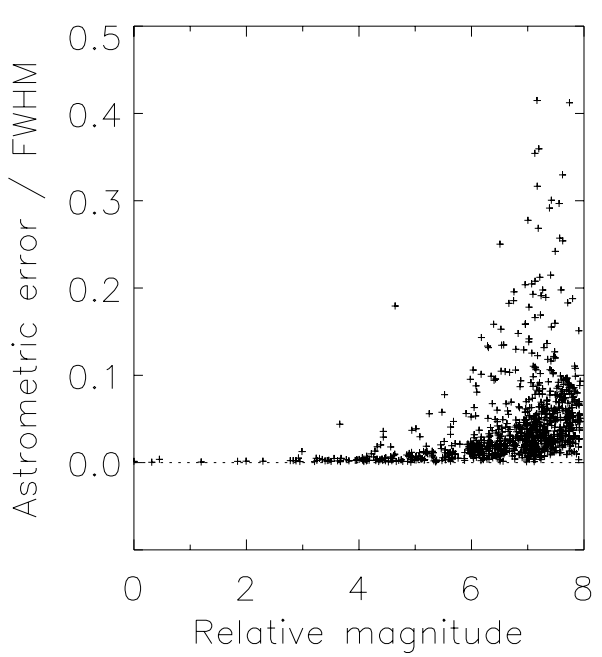

Fig. 7. Plot of astrometric errors vs. relative magnitude of detected stars; the errors are quoted in FWHM units (1 FWHM $\sim 3.6$ pixel) and represent the distance between the calculated and the true position. A tolerance of 1 FWHM has been chosen to find the coincidences between the detected stars and their true counterparts. The small errors for the brightest stars are due to blending effects

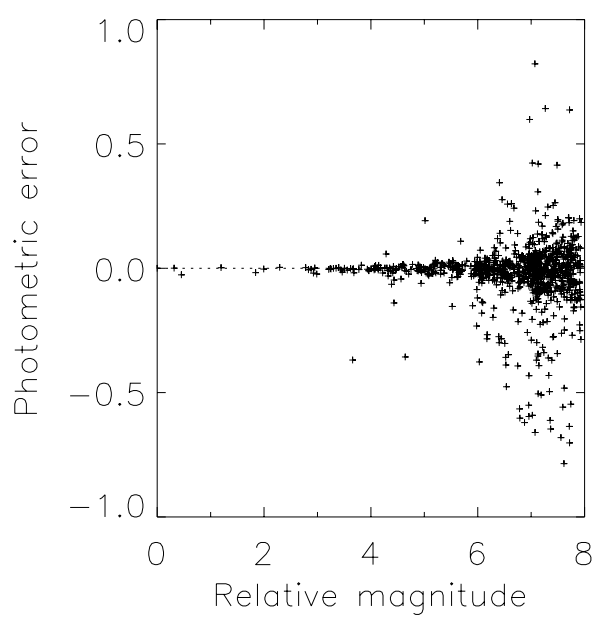

Fig. 8. Plot of photometric errors vs. relative magnitude of detected stars. The brightest star in the field has mag $=0$ by definition

0.1 magnitudes. The stars with less accurate astrometry or photometry are generally faint: $\sim 75 \%$ belong to the magnitude interval from 7 to $8, \sim 20 \%$ to the bin between magnitude 6 and 7 and only $\sim 5 \%$ are distributed in lower bins of the luminosity function. These stars have, in $\sim 45 \%$ of the cases, a lost source in their immediate neighborhood within the first diffraction ring of the PSF. The others are faint stars dispersed in the halo of the brightest sources or in the noisy background nebulosity.

After discussing the performance of the code with a standard analysis, it is interesting to examine how the results are affected by the main parameters of the method. Applying the de-blending strategy we detected $\sim+10 \%$ 


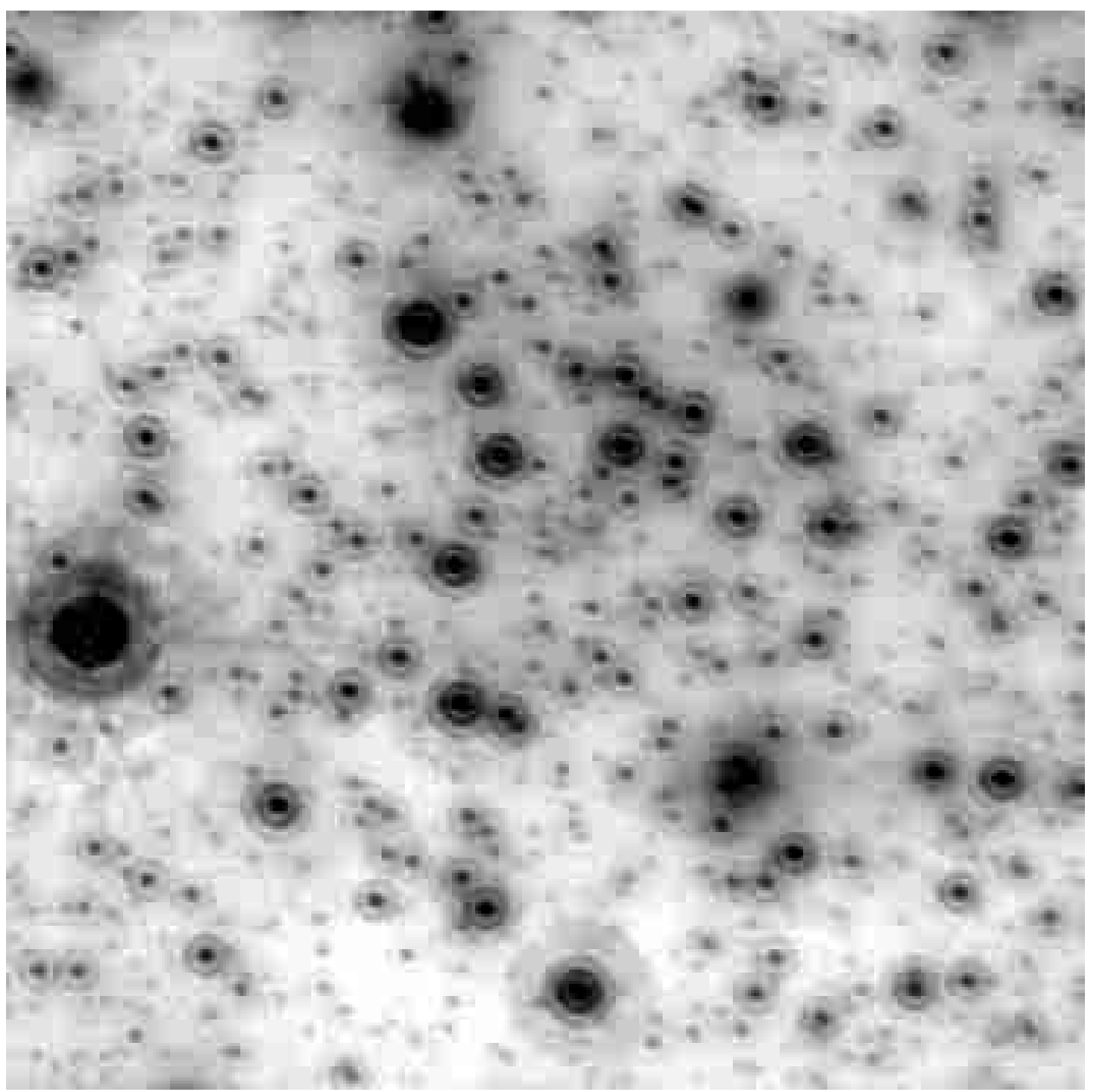

Fig. 9. PUEO image of the Galactic Center. North is to the left (at an angle of $-100.6^{\circ}$ from vertical) and east is $-10.6^{\circ}$ from the vertical. The display stretch is logarithmic

more binaries in the separation range between $1 / 2$ and 1 PSF FWHM, even though the overall detection gain is less than $1 \%$ referred to the total number of sources. With a higher number of iterations of the main loop we detected $\sim+30 \%$ more binaries in the range from 1 to 2 PSF FWHM. Decreasing the detection threshold from 3 to 1 times the noise standard deviation, we found $\sim+25 \%$ more binaries in the range between $1 / 2$ and $1 \mathrm{PSF}$ FWHM, but with 10 faint $(\mathrm{mag}>8)$ false detections instead of 1 . Increasing the threshold on the correlation coefficient, from 0.7 to 0.8 , we reported no false detection, but the number of lost stars increased by about $60 \%$; the additional lost sources were generally fainter than magnitude 7 , but not necessarily in crowded groups. Lowering the correlation threshold to 0.6 we detected more faint isolated stars and binaries, at separations between 1 and 2 PSF FWHM, but with a higher probability of false detections (2 instead of 1 ). Finally the astrometric and photometric accuracy approaches a stable level after a few $(\sim 2)$ re-fitting iterations.

\subsection{Galactic center}

The code was run on a 15 min exposure time $K$ band $(2.2 \mu \mathrm{m})$ image of the Galactic Center (Fig. 9), taken with the PUEO AO system on the $3.6 \mathrm{~m} \mathrm{CFH}$ telescope (Rigaut et al. 1998). The Strehl ratio in the image is $\sim 45 \%$. The PSF FWHM is $\sim 0.13^{\prime \prime}$, with a sampling of $0.034^{\prime \prime} /$ pixel. The Adaptive Optics guide star was a $m_{\mathrm{R}}=14.5$ star (called star 2 in Biretta et al. 1982) located about (to the upper left) $20^{\prime \prime}$ from the center of the image, out of the field of view of the figure. There is therefore a slightly elongation of the PSF towards the direction of the guide 


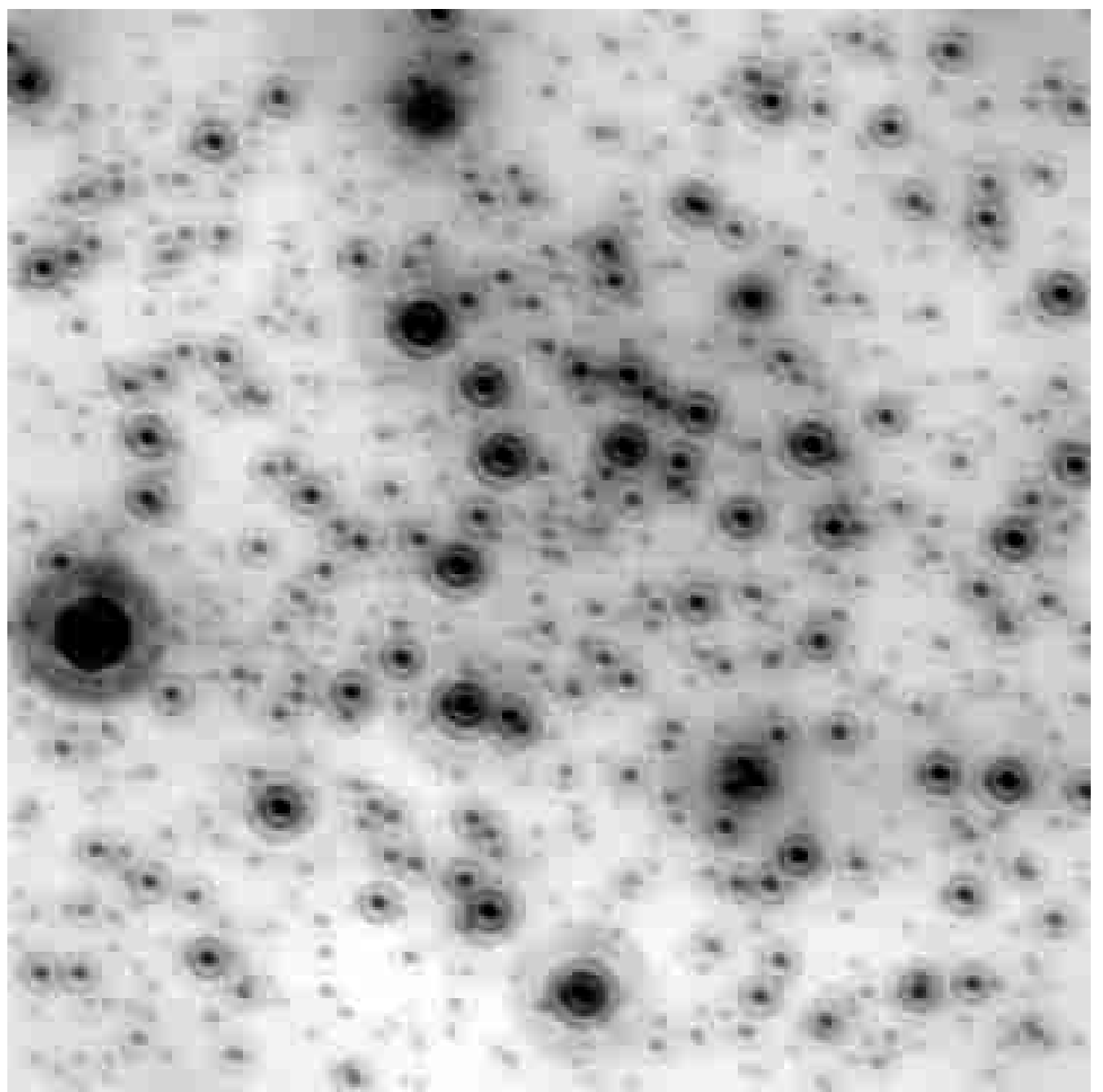

Fig. 10. Reconstructed image, given by the sum of about 1000 detected stars and the estimated background. The display stretch is logarithmic

star. However, due to the fact that the isoplanatic patch was much larger than the $13^{\prime \prime} \times 13^{\prime \prime}$ shown in the figure, a space-invariant PSF fits the data very well, as we will show.

A standard analysis was performed, analogous to the one described in Sect. 4.1 for the synthetic stellar field. About 1000 stars were detected, with a correlation coefficient of at least 0.7 ; the reconstructed image is shown in Fig. 10.

We evaluated the accuracy of the algorithm by means of an experiment with synthetic stars. We created 10 frames adding to the original image $10 \%$ of synthetic stars at random positions for each magnitude bin of the estimated luminosity function. The 10 frames were analyzed separately. As in the simulated case, a distance tolerance of 1 PSF FWHM was adopted to find coincidences between the detected stars and their true counterparts. The lists of detected artificial stars were merged together and the astrometric and photometric errors were computed and plotted as a function of the true magnitude (Figs. 11 and 12).

The plots show no apparent photometric bias and high astrometric and photometric accuracy: the stars brighter than magnitude 5 , for instance, have a mean astrometric error $<0.5$ mas and a mean absolute photometric error $<0.01 \mathrm{mag}$. It should be stressed however that the artificial sources are contaminated by the background noise present in the observed data and by the photon noise due to neighboring stars, but no additional noise was added. Figure 13 shows a comparison between the mean luminosity function retrieved in the 10 experiments and the truth. Assuming an expected error for each bin equal to the square root of the corresponding number of counts, according to the Poisson statistic, the only significant differences occur for magnitudes fainter than 9. It is also interesting to consider the magnitude distribution of the false detection cases (dashed-dotted line in Fig. 13), i.e. the detected stars for which we found no counterpart in the original list, within a distance of 1 PSF FWHM. 


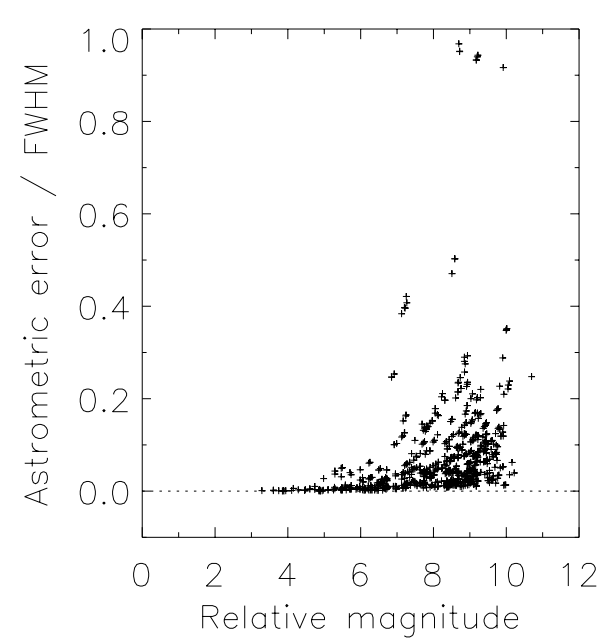

Fig. 11. Plot of astrometric errors vs. relative magnitude of detected synthetic stars; the errors are quoted in FWHM units ( 1 FWHM $\sim 4$ pixel) and represent the distance between the calculated and the true position

The mean percentage of false detections in the 10 experiments is $2 \%$ of the total number of stars. The false detections are almost always very faint $(\mathrm{mag}>8)$; their number is comparable to the square root of the total counts only in the last magnitude bin, for magnitudes fainter than 10 . The percentage of false detections reported in these experiments seems to confirm the analysis performed by visual inspection on the stars detected in the original frame.

\section{The StarFinder code}

The StarFinder code has been provided with a collection of auxiliary routines for data visualization and basic image processing, in order to allow the user to analyze a stellar field, produce an output list of objects and compare different lists, e.g. referred to different observations of the same target. The input image is supposed to be just calibrated.

The code is entirely written in the IDL language and has been tested on Windows and Unix platforms supporting IDL v. 5.0 or later. A widget-based graphical user interface has been created. The main widget appearing on the computer screen is nothing more than an interface to call secondary widget-based applications, in order to perform various operations on the image. The basic documentation about the code can be found in the on-line help pages. IDL users might wish to run interactively the StarFinder routines, without the widget facilities: complete documentation on each module is available for this purpose.

\section{Conclusions and future developments}

The elaboration of real and simulated data seems to prove the effectiveness of StarFinder in analyzing crowded isoplanatic stellar fields characterized by high Strehl ratio

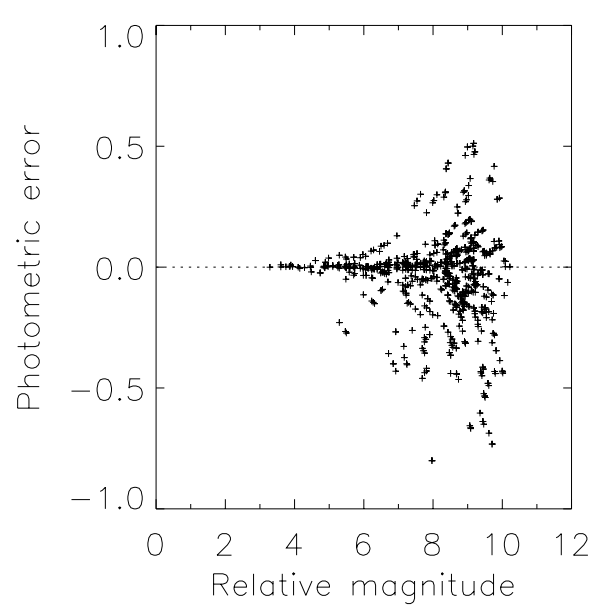

Fig. 12. Plot of photometric errors vs. relative magnitude of detected synthetic stars. The brightest star in the field has mag $=0$ by definition

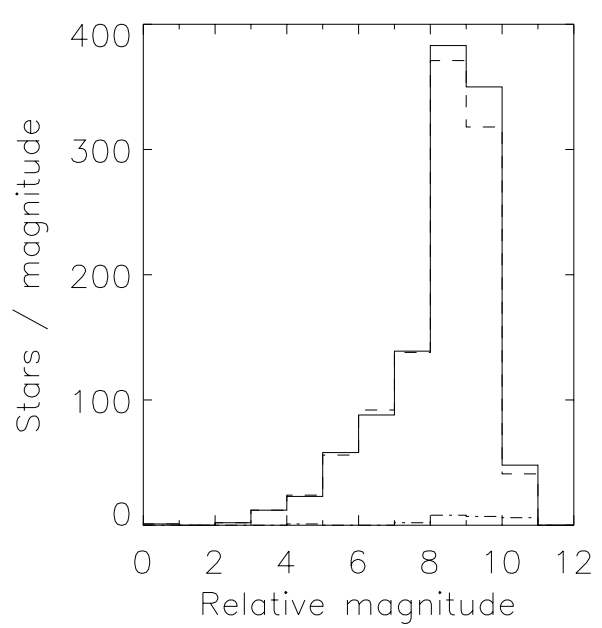

Fig. 13. Luminosity function after adding synthetic stars (continuous line) compared to the mean luminosity function resulting from the analysis of the 10 frames with artificial sources (dashed line); the dotted-dashed line indicates the mean luminosity function of the false detections

PSFs and correct sampling, reaching in this case the full utilization of the data information content. The code can be applied also to low Strehl data with results comparable to those attainable by other methods. Moreover we are evaluating its performance on undersampled images.

StarFinder is also reasonably fast: the analysis of the Galactic Center image $(368 \times 368$ pixels, $\sim 1000$ stars $)$ requires between 5 and 10 minutes on a normal PC (Pentium Pro $-64 \mathrm{Mb}$ RAM $-350 \mathrm{MHz}$ ). The graphic interface makes it accessible to users unfamiliar with IDL.

In the near future we plan to provide the code with tools to handle images with spatially variable PSF and new elements which might be helpful in the analysis of a stellar field. An interesting application, still in progress, aims at cleaning mixed fields from the contamination 
of foreground stellar images, leaving out true or suspected diffuse sources.

The StarFinder package and its technical documentation are directly available at: http://www.bo.astro.it or can be obtained on request to E. Diolaiti.

Acknowledgements. François Rigaut is thanked for kindly providing the PUEO image of the Galactic Center and for supporting the initial development of this method. We acknowledge the support of the ESO La Silla $3.6 \mathrm{~m}$ telescope team during the technical observations with Adonis, from which we have retrieved "real" PSFs used to simulate stellar fields. This work was partially supported by the ESO Instrumentation Division Adaptive Optics Dept. and by the Italian Ministry for University and Research (MURST) under grant Cofin 9802-32.

\section{References}

Almoznino E., Loinger F., Brosch N., 1993, MNRAS 265, 641

Aloisi A., et al., 2000 (in preparation)

Beck J.V., Arnold K.J., 1977, Parameter estimation in engineering and science. John Wiley \& Sons

Bendinelli O., Parmeggiani G., Piccioni A., Zavatti F., 1987, AJ 94, 1095

Bertin E., Arnouts S., 1996, A\&AS 117, 393

Bevington P.R., Robinson D.K., 1994, Data reduction and error analysis for the physical sciences. McGraw-Hill

Bijaoui A., 1980, A\&A 84, 81

Biretta J.A., Lo K.Y., Young P.J., 1982, ApJ 262, 578

Currie D.G., et al., 2000a, The ESO Messenger 100, 6

Currie D.G., et al., 2000b, SPIE 4007, 866

Christou J.C., Bonaccini D., 1996, in: Technical Report ESO VLT Doc., No. GEN-TRE-ESO-11620-1261

Diolaiti E., Bendinelli O., Bonaccini D., Parmeggiani G., Rigaut F., 1999, in: Proc. of the ESO/OSA Meeting on Astronomy with Adaptive Optics, Bonaccini D. (ed.). ESO Garching, p. 175
Diolaiti E., Bendinelli O., Bonaccini D., Close L.M., Currie D.G., Parmeggiani G., 2000a, in: Proceedings of ADASS IX, Crabtree D., Manset N., Veillet C. (eds.), ASP Conf. Ser. San Francisco (in press)

Diolaiti E., Bendinelli O., Bonaccini D., Close L.M., Currie D.G., Parmeggiani G., 2000b, SPIE 4007, 879

Esslinger O., Edmunds M.G., 1998, A\&AS 129, 617

Fruchter A.S., Hook R.N., Busko I.C., Mutchler M., 1997, in: HST Calibration Workshop, STScI, Casertano S. et al. (eds.)

Fusco T., Conan J.-M., Mugnier L.M., Michau V., Rousset G., 2000, A\&AS 142, 149

Gonzalez R.C., Woods R.E., 1992, Digital image processing. Addison-Wesley

Krist J., Hook R., 1999, Tiny Tim User's Manual Ver.5.0, Space Telescope Science Institute, Baltimore

Landsman W.B., 1995, in: Proceedings of ADASS IV, Shaw R.A., Payne H.E., Hayes J.J.E. (eds.), ASP Conf. Ser. San Francisco 77, 437

Lorenzutta S., 1967, in: CNEN Report Doc. Cec. (67), No. 16

Luenberger D.G., 1984, Linear and nonlinear programming. Addison-Wesley Publishing Company

Mariotti J.-M., 1988, in: Proc. NATO Advanced Studies Institute on "Diffraction-limited imaging with very large telescopes", NATO ASI Series, Series C: Math. Phys. Sci., Alloin D.M., Mariotti J.-M. (eds.), p. 3

Park S., Schowengerdt R., 1983, Computer Vision, Graphics Image Proc. 23, 256

Rigaut F., Salmon D., Arsenault R., Thomas J., Lai O., Rouan D., Véran J.-P., Gigan P., et al., 1998, PASP 110, 152

Rust B.W., Burrus W.R., 1972, Mathematical programming and the numerical solution of linear equations. American Elsevier Publishing Company

Stetson P.B., 1987, PASP 99, 191

Véran J.-P., Rigaut F., Maitre H., Rouan D., 1997, J. Opt. Soc. Am. A 14, No. 11, 3057

Véran J.-P., Rigaut F., 1998, SPIE 3353, 426

Yaroslavsky L.P., 1997, Appl. Opt. 36, No. 2, 460 